\title{
Axe 1 : Sociohistoire de l'État social depuis 1945 - mutations, européanisations et capacités
}

\section{Karim Fertikh et Heike Wieters}

\section{OpenEdition}

\section{Journals}

Édition électronique

URL : http://journals.openedition.org/ifha/7985

DOI : 10.4000/ifha.7985

ISSN : 2198-8943

\section{Éditeur}

IFRA - Institut franco-allemand (sciences historiques et sociales)

\section{Édition imprimée}

Date de publication : 31 décembre 2014

ISSN : 2190-0078

\section{Référence électronique}

Karim Fertikh et Heike Wieters, «Axe 1 : Sociohistoire de l'État social depuis 1945 - mutations, européanisations et capacités », Revue de l'IFHA [En ligne], 6 | 2014, mis en ligne le 31 décembre 2014, consulté le 19 avril 2019. URL : http://journals.openedition.org/ifha/7985 ; DOI : 10.4000/ifha.7985

Ce document a été généré automatiquement le 19 avril 2019

(CIFHA 


\title{
Axe 1 : Sociohistoire de l'État social depuis 1945 - mutations, européanisations et capacités
}

\author{
Karim Fertikh et Heike Wieters
}

\section{NOTE DE L'ÉDITEUR}

En collaboration avec les membres du groupe de recherche «État social », en particulier : Hadrien CLOUET, Gabriel COLLETIS, Arnaud LECHEVALIER, Gabriele METZLER, Jay ROWELL, Berthold VOGEL, Michael WERNER et Bénédicte ZIMMERMANN.

1 Le groupe étudie le développement des États-providence après 1945 en accordant une attention particulière aux processus d'institutionnalisation et aux acteurs sociaux de cette institutionnalisation. La fin de la Seconde Guerre mondiale, le début et la fin du conflit Est-Ouest, la construction européenne tout comme les changements économiques, politiques, sociaux et culturels ont marqué les États sociaux européens de manière significative ${ }^{1}$. Les transformations dans le domaine des politiques sociales, de l'éducation et du marché du travail ainsi qu'en matière d'assurance vieillesse et santé (en résumé la prise en charge collective de risques considérés comme sociaux par des institutions privées ou publiques) ${ }^{2}$ ont eu et exercent encore des conséquences étendues sur le fonctionnement, sur la perception et la légitimité dans l'espace public des institutions étatiques et supra-étatiques ${ }^{3}$. Le processus de l'intégration économique européenne renvoie ainsi directement à la question d'une "européanisation" des phénomènes sociaux et des dynamiques institutionnelles et à la non-simultanéité des changements économiques, politiques, sociaux et culturels en Europe.

2 Ce n'est cependant pas une comparaison ou une classification des systèmes nationaux des États-providence européens ${ }^{4}$ qui constitue le cœur du travail de recherche du groupe, mais l'étude des réseaux d'acteurs, des interdépendances et des processus de transfert au- 
delà des frontières nationales ou systémiques ${ }^{5}$. Avec le changement des institutions nationales et « européennes » de l'État-providence, les attentes et les possibilités d'action des sujets concernés se modifient elles aussi - que ce soit celles des citoyens de l'Union européenne ou celles des "Non-Européens». Le changement institutionnel est directement corrélé aux chances de participation à la vie sociale et politique, aux chances de réalisation de soi des différents sujets et à leur comportement envers les instruments bureaucratiques et les injonctions de nature étatique et supra-étatique. Ce changement ne peut s'expliquer sans adopter un regard différencié sur les acteurs, et leurs actions à l'intérieur (et à l'extérieur) de ces institutions ${ }^{6}$. Il s'agit donc de comprendre et d'expliquer non seulement les grands mouvements et les processus à long terme mais aussi de repérer les "scènes $»^{7}$ tout à fait concrètes sur lesquelles les acteurs produisent de (nouvelles) catégories d'action ou se les approprient et négocient les chances ou les limites de leur action individuelle. En adoptant une perspective issue des sciences économiques, sociales et historiques, le groupe de recherche "État social " porte par conséquent une attention particulière aux processus d'institutionnalisation dépassant les systèmes à l'échelle microsociale. Plus qu'à un état des lieux, le réseau thématique s'intéresse aux processus de construction de ces institutions, à leur appropriation quotidienne par les individus, à leurs effets sur ces derniers, ainsi qu'aux compromis et aux règles du jeu grâce auxquels les acteurs concernés peuvent instaurer et influencer ces institutions. Le groupe de travail analysera en conséquence la production, l'usage et les effets des institutions en tant qu'unité.

Pour ce faire, il est possible de poursuivre le travail à partir de résultats déjà existants. Les systèmes sociaux en Europe font déjà depuis longtemps l'objet d'un intérêt étendu et interdisciplinaire - la composition du groupe de travail qui comprend de nombreux experts renommés dans le domaine de la recherche sur l'État social le prouve. En se fondant sur ce savoir, il est possible d'identifier de nouveaux champs de relations, qui, dans le cadre du réseau thématique ici proposé, doivent être travaillés et, dans la mesure du possible, rendus productifs.

Le groupe de travail se penchera ainsi sur quatre complexes thématiques (relations intergénérationnelles et démographie; (in)égalités; savoir et statistique; travail) en s'appuyant sur trois types de méthodologie transversale (européanisation; sociohistoire ; sociologie et analyse des capabilités/capacités). Il pourra être fait recours à des travaux antérieurs dont les thèmes et les problématiques devront être cependant élargis et mis en rapport entre eux de manière innovante. Pour ces six domaines, il s'agit de thèmes et d'approches qui, en les combinant, sont propres à produire des effets de synergie de la recherche et à générer de nouveaux éléments de connaissance. Les trois approches méthodologiques se situent à la croisée des disciplines et rendent ainsi le dialogue possible entre économistes des institutions, historiens, politistes et sociologues. 


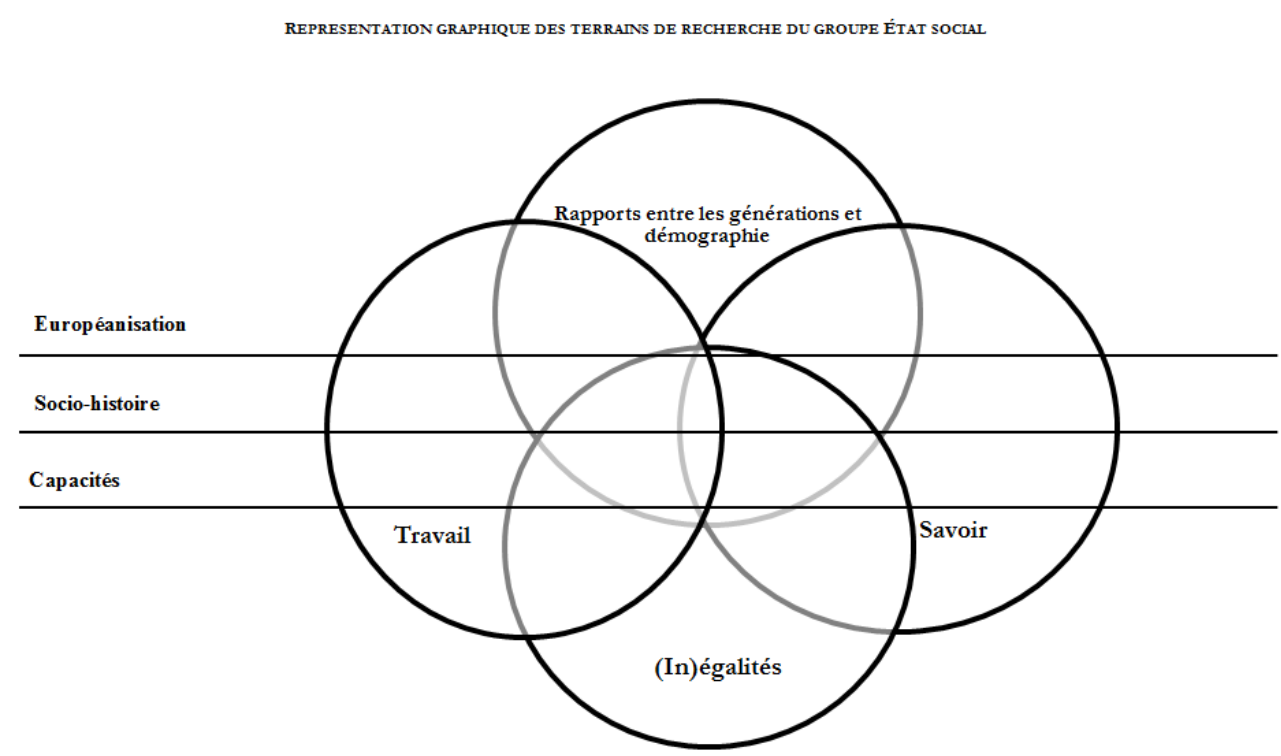

\section{Domaines thématiques}

Les domaines cités ci-dessus vont être brièvement esquissés et mis en relation afin d'expliciter le programme de travail envisagé par le réseau thématique :

\section{a) Relations intergénérationnelles et démographie}

6 Alors qu'il existe déjà en Allemagne des études sur les dimensions structurelles, normatives et culturelles des relations intergénérationnelles dans les (sub)systèmes de l'État-providence - surtout en ce qui concerne l'assurance vieillesse ${ }^{8}$ - ce complexe thématique a tendance jusqu'à présent à être sous représenté dans la recherche française. S'il est question en Allemagne, surtout en ce qui concerne les modèles d'assurance vieillesse par répartition, de la "génération comme argument ", dans le contexte français les questions de participation et de représentation en politique dominent jusqu'à présent, points qui, inversement, sont souvent négligés dans le débat allemand. Même si, en France aussi, il est accordé une grande importance aux interrogations structurelles et normatives ${ }^{10}$, les deux traditions de recherche ne se laissent jusqu'à présent que difficilement comparer. La communication scientifique, sans parler d'une recherche commune dans un contexte franco-allemand ou même européen, est par conséquent à peine existante au sujet des relations intergénérationnelles, des transferts et des concepts normatifs. Au vu du changement permanent des tendances démographiques en Europe, lequel engendre des effets de plus en plus visibles sur les institutions sociales et politiques ${ }^{11}$, un approfondissement du débat scientifique sur les relations intergénérationnelles semble s'imposer. Dans le cadre du réseau thématique doivent être discutés tout autant le changement des relations intergénérationnelles au sein et au travers du processus d'intégration européenne au niveau des politiques communes de l'assurance vieillesse et de l'éducation (cf. projet de recherche de Heike Wieters) que l'établissement de catégories sociales et politico-administratives (comme par exemple celle de la "dépendance», cf. projet de recherche de Karim Fertikh ${ }^{12}$ en matière de service public. Les approches sociohistoriques (cf. méthodes, point 2) qui interrogent les catégories d'action, les acteurs et 
les lieux où ces catégories sont négociées ${ }^{13}$ et les perspectives de recherche, qui font des chances de réalisation de soi (cf. méthodes, point 3) des "vieux» et des "jeunes » Européennes et Européens au sein de systèmes sociaux-étatiques en cours de transformation un point central, joueront un rôle important.

\section{b) Égalité \& inégalité}

7 Les institutions et les mesures sociales-étatiques prises ne deviennent un objet véritable de recherche qu'au vu de leur inscription dans leurs contextes spécifiques interventionnistes, de droit politique et normatif en matière de réforme sociale ${ }^{14}$. Cependant, le rapport social à l'égalité et à l'inégalité socio-économiques, politiques et normatives se laisse décrire comme un dispositif fonctionnel commun et fondamental de l'État-providence ${ }^{15}$. La question de savoir quel rôle l'(in)égalité joue pour la perception et l'analyse de la réalité sociale ne va pas de soi. Déterminer si l'inégalité est un mal social à dépasser (par l'examen et la mesure) ou si elle se situe au fondement de la complexité de la société, de la mobilité et de l'innovation, reste une tâche sujette à discussion ${ }^{16}$. Un groupe de travail interdisciplinaire peut partir d'un tel constat. En effet, le changement dans le rapport social à l'égalité et à l'inégalité en Europe se laisse appréhender sur un plan normatif et politique ${ }^{17}$ mais il se laisse aussi analyser en terme de structures, de questions de répartition ${ }^{18}$, de chances d'accès, de manières de se subjectiver (cf. Point 6). Les questions consistant à savoir où, comment et à quel niveau les structures étatiques et les institutions de l'État-providence ne font pas que maintenir l'inégalité mais en produisent aussi une nouvelle, restent dans beaucoup de domaines (et notamment dans le domaine des relations intergénérationnelles dans le contexte européen) toujours ouvertes $^{19}$. Cela vaut donc la peine d'observer plus précisément les faits au niveau des sujets et de leurs pratiques et de considérer leur rapport à l'inégalité économique, sociale ou politique, leur rapport aux mécanismes d'inclusion et d'exclusion. La question de savoir si (et si oui comment et où exactement) l'« Europe » devient le nouvel espace de référence à l'intérieur duquel les (in)égalités dans le domaine de l'État-providence sont discutées, renforcées ou nivelées, se situe donc directement au centre de l'attention du groupe de travail.

\section{c) Savoirs et statistiques}

8 L'État social existe par le travail qu'accomplissent au quotidien ses agents pour formuler des catégories et pour convertir les situations singulières en pensée d'état nomenclaturée. Les interrogations sur les constructions savantes de l'État social, portant à la fois sur les catégories de politique publique et sur la professionnalisation des experts d'État, ont conduit à de nombreux travaux en sciences humaines et sociales ${ }^{20}$. Le mot d'ordre historiographique de la "scientifisation du social ${ }^{21}$ a montré sa fécondité heuristique sur les cas nationaux ${ }^{22}$. L'intégration européenne met les sciences sociales au défi de sortir des « containers nationaux ${ }^{23}$ pour penser les diverses formes d'articulation des espaces locaux, nationaux et européens à partir d'une perspective centrée sur les acteurs et les processus d'institutionnalisation. Parmi les pistes de recherche de notre groupe de travail, trois interrogations méritent d'être ici évoquées. D'abord, la question de l'européanisation des savoirs d'État et la construction d'un espace européen de l'expertise peut être pensée sur le mode de la mobilisation de ressources proprement européennes ${ }^{24}$ permise par la construction d'un faisceau d'organisations supra-étatiques. 
En effet, la constitution d'un ensemble d'institutions européennes est le vecteur de construction d'une demande publique d'expertise : à travers la commande de rapports ou d'expertises par les institutions européennes, à travers la mise en place d'administrations de production de savoirs sur l'Europe, à l'instar d'Eurostat (dont Anne Lammers est la première à proposer d'écrire l'histoire) ou à travers les stratégies d'influence appuyées sur l'expertise via les think tanks ou les lobbys obligeant à la production de savoirs et de savoir-faire européens ${ }^{25}$. Ces ressources proprement européennes ne peuvent être ignorées tant que l'optique est de comprendre la synchronisation des temporalités nationales des réformes de l'État social ou celle de leur convergence. Ensuite, l'européanisation des politiques sociales peut être explorée à travers la circulation des experts nationaux et des catégories d'action publique en Europe, notamment à travers les références aux « modèles » nationaux - danois, allemand, britannique etc. (cf. : projet de thèse d'Hadrien Clouet sur le chômage partiel). Ce sont là les configurations d'acteurs qui permettent l'objectivation de savoirs européens, dont les effets en retour sur les champs politiques nationaux sont à analyser, qui sont visées par nos recherches. L'existence de ce phénomène n'est pas nouvelle, puisque la concurrence entre les États nationaux au XIXe siècle avait déjà conduit à une circulation européenne des politiques sociales ${ }^{26}$ mais l'intensité des échanges et l'importance de leurs effets en matière de convergence des politiques sociales peuvent faire l'objet d'une étude. L'étude des experts européens doit mettre en évidence les sources du "crédit» social des experts. Les modalités de reconnaissance des experts et des ressources pertinentes (crédit universitaires positions institutionnelles nationales, participation à des organisations non gouvernementales internationales etc.) constituent l'une des questions qu'on se posera (cf. projet de thèse d'Hugo Canihac et de Mariette Fink).

Les questions des statistiques, comme constitutives de la réalité sociale qu'elles décrivent ${ }^{27}$ sont aussi centrales dans nos interrogations. Ces constructions statistiques obligent à mettre en place des arrangements pratiques permettant de décrire pour des fins de gestion administrative les populations européennes ${ }^{28}$ (cf. projets de recherche d'Anne Lammers et d'Alice Lavabre). La construction de cet outillage mental est donc un vecteur important dans l'européanisation de certains problèmes et de certains instruments de politique publique.

\section{d) Travail}

10 Les mobilisations autour de la "question sociale", centralement l'ensemble des problèmes construits par les porte-parole du salariat en constitution, ont joué un rôle central dans l'émergence de l'État social en Europe ${ }^{29}$. L'État providence prend appui sur les formes hétérogènes de solidarité et d'organisation collectives du travail pour assurer un champ de protection basé sur des catégories devenant génériques (accident du travail, maternité, retraite, maladie, chômage), qui se transforment au fil du temps ${ }^{30}$ (cf. projet de recherche de Sarah Hasdenteufel). La couverture de ce qui se définit comme risque s'est donc construite dans le champ du travail à travers des logiques d'intervention et de catégorisation plurielles.

11 La « nouvelle question sociale » diagnostiquée par Robert Castel a pour effet d'ébranler ce modèle de solidarité, et permettre à de nouvelles catégories d'intervention d'apparaître : la «sécurisation du parcours professionnel » individuel prend la place de la protection collective de et dans l'emploi, l'incitation au travail celle de la lutte contre le chômage. Ce 
faisant, de multiples rapports au travail se mettent en place, et mettent aux défis les formes instituées de sécurité sociale: les formes précaires d'emplois, les secteurs d'emploi à bas salaire, les effets de halo et les nouvelles pratiques de catégorisation des chômeurs dans les agences d'emploi ${ }^{31}$. Ces diverses formes d'« activation» des politiques d'emploi et la pluralisation des formes d'emploi, de sous-emploi et de chômage en Europe constituent dès lors un observatoire des transformations des sociétés européennes et des arrangements institutionnels de leurs systèmes de solidarité.

Elles complexifient les rapports que les bénéficiaires entretiennent avec les agents de l'État ou des agences publiques ou privées en charge de l'exécution des politiques relatives au travail ${ }^{32}$ (cf. projet de recherche d'Hadrien Clouet). Elles soulèvent les questions des capacités d'appropriation active des nouveaux «droits» (droits à la formation, politiques de lutte contre les discriminations, en particulier de genre) et des injonctions institutionnelles par les individus auxquels elles s'adressent ${ }^{33}$. L'intérêt de notre groupe de recherche ne porte pas uniquement sur la comparaison des systèmes nationaux, considérés comme des ensembles, mais sur des processus de transferts et de croisement dans le développement des institutions et la construction du niveau européen comme échelle pertinente de l'action. Loin de considérer ces éléments comme ressortissant de pures logiques individuelles, notre groupe de travail entend considérer les logiques collectives de formation des identités au travail et au chômage (cf. projets de recherche de Nicolas Briot et Hadrien Clouet), de fabrication/démantèlement et d'appropriations des droits. L'européanisation se fait particulièrement sentir dans la construction et la diffusion de politiques de lutte contre les discriminations, en particulier de genre et de gendermainstreaming (cf. projet de recherche de Mariette Fink). L'ensemble de ces interrelations complexes, entre agents et bénéficiaires, entre le salarié, son entreprise et les politiques publiques, ainsi que la fabrication et l'usage des catégories - avec leurs appropriations par les individus - constituent donc un des terrains de recherche de notre équipe.

\section{Approches méthodologiques}

\section{a) Européanisation}

13 Le concept d'européanisation a une longue tradition en sciences sociales et politiques. Dans ces disciplines, il est employé comme un concept à la fois descriptif et analytique qui permet d'appréhender et de décrire les nouvelles formes des processus de gouvernance et d'institutionnalisation purement européens (surtout à partir des années 1990). Ces dernières années, ce concept a de plus en plus été utilisé également en histoire, même si, en même temps les connotations à la fois normatives et téléologiques que pouvaient porter ce concept en sciences sociales étaient critiquées et remises en cause ${ }^{34}$. L'européanisation n'est donc ni une voie à sens unique qui mènerait nécessairement à une Europe unie, ni un concept qui permettrait de déterminer sans problème des objets ou des lieux d'analyse prédestinés qui rendraient possible une mise en évidence et une analyse de l'européanisation comme un processus se déroulant de manière linéaire. L'existence de formes européennes en transformation ou de nouvelles formes européennes de référence, d'unification ou de transmissions des compétences peut être prouvée aux niveaux tant politique, social et culturel que juridique et économique - à chaque fois en tenant compte des structures et des institutions tout comme des idées, des 
concepts et des pratiques quotidiennes. D'autre part, des mouvements opposés, une certaine dissociation ou un certain repli sur les traditions nationales ou régionales, sur les institutions ou les ressources deviennent de plus en plus visibles. Il est donc nécessaire de penser l'européanisation de manière multidimensionnelle tant dans une perspective spatiale que temporelle: différents processus d'européanisation peuvent se dérouler en parallèle ou à l'opposé, en se croisant voire s'interrompant ou de manière totalement asynchrone, comme le montre par exemple la disparité actuelle entre l'intégration économique et politique au sein de l'Union européenne. Cette constatation vaut aussi et surtout pour les institutions en charge de la sécurité sociale en Europe. Alors que certains domaines proches de l'économie ont été harmonisés dès l'époque de la CECA et ont été européanisés institutionnellement et juridiquement, comme par exemple le réajustement au niveau européen des montants de l'assurance vieillesse découlant de la libre circulation des travailleurs; d'autres domaines et institutions sont soumis à d'autres logiques. Les systèmes de l'État-providence, toujours surdéterminés dans leur essence nationalement, se retrouvent néanmoins de plus en plus en compétition au niveau institutionnel. Dans cette compétition, où l'Union européenne fait office de ressource (aussi financière) et d'espace de référence, naissent lentement des politiques sociales purement européennes ${ }^{35}$. Le groupe de travail placera ici surtout au centre de son travail les processus complexes d'apprentissage et de transfert que les experts, les conseillers économiques et politiques européens ${ }^{36}$ tout comme les échanges institutionnels directs, les procédés de plus en plus standardisés (souvent plus «internationaux" qu'« européens ») et les techniques de management ont fait progresser.

\section{b) Socio-histoire}

14 «Les concepts de société, de communauté solidaire et les catégories juridiques de souveraineté ou de principe de l'égalité de traitement sont historiquement mais aussi conceptuellement en rapport avec les évolutions de l'État-nation. [...] L'européanisation des relations sociales et des processus politiques mène cependant à des possibilités d'action et à des définitions des problèmes autres [...et] exige une redéfinition des concepts et des catégories qui tienne compte de la genèse et des effets des possibilités d'actions et des définitions de problèmes ancrées au niveau de l'État-nation. $»^{37}$. Ce constat est particulièrement valable pour le thème général du groupe de travail et pour le réseau thématique "État-social», pour lequel une aide est ici sollicitée, dont la terminologie changeante et les instruments semblent être faits pour une analyse adoptant l'approche de la socio-histoire, développée dès les années 1980 en France $^{38}$. Cette perspective repose sur une volonté de dénaturaliser les institutions sociales telles qu'elles ont été objectivées et réifiées dans les interactions ${ }^{39}$. La socio-histoire essaie de déplacer le regard des grandes idées et des grands récits macrosociaux vers des analyses des rapports sociaux concrets pour ainsi étudier conflits, processus de négociation et glissements de sens dans le domaine de l'action et des catégories sociales. Elle a, en ce sens, plusieurs intérêts méthodologiques: rappeler les significations que les catégorisations et les instruments de politique sociale ont dans les divers moments où ils sont forgés, rappeler que les politiques sociales sont le résultat de compromis et de rapports de force au sein des administrations et entre les administrations et divers secteurs de la société, rappeler que les appropriations de ces politiques peuvent en déplacer le sens et enfin montrer la manière dont «le mort saisit le vif » et le rôle des catégories vécues et des institutions dans la formation du monde vécu. De cette manière, 
le niveau des acteurs, y compris d'un point de vue prosopographique, est replacé au centre de l'intérêt. Par une attention résolument historique et sociologique portée sur le contexte de création, sur les glissements de sens comme sur les effets à long terme de nouvelles pratiques et catégories dans les interactions concrètes entre les différents acteurs (bureaucrates et citoyens, "conseillers pour l'emploi» et «bénéficiaires de prestations sociales », etc.) les processus de transformations, qui ont touché les Étatssociaux européens et leurs citoyennes et citoyens, sont rendus alors concrets et visibles. Les intérêts méthodologiques sont divers: premièrement, il s'agit de saisir les significations et les catégorisations des instruments sociopolitiques au moment de leur création et pendant leur évolution au cours du temps; deuxièmement il faut se souvenir de ces instruments et de ces institutions comme étant le résultat de compromis sociaux et de processus de négociation. En conclusion, l'influence et les effets de ces catégories et de ces institutions sur le quotidien des sujets peuvent être ainsi analysés.

\section{c) Sociologie et approche par les capabilités}

15 L'approche par les capabilités, développée dès le début des années 1980 par Amartya Sen en coopération avec Martha Nussbaum, s'intéresse à l'élargissement et à la modification de concepts traditionnels mesurant le bien-être individuel et collectif ${ }^{40}$. En critiquant l'unidimensionnalité du PIB et des données sur les revenus comme indicateurs de bienêtre (well-being) et de développement social et en développant avec le concept de «capabilités » ou de «chances de réalisation de soi » une nomenclature alternative, Sen et Nussbaum élargissent la compréhension des actions individuelles et sociales en matière d'économie : non seulement la rationalité finaliste et les motifs utilitaristes mais aussi les liens sociaux, les possibilités d'action différenciées et les degrés (subjectifs) de liberté deviennent non seulement des critères de "vie bonne", mais aussi des critères économiques pertinents. Cette conception issue des sciences économiques a été reprise par des membres du groupe de travail qui lui ont donné par la suite une nouvelle orientation ${ }^{41}$. Cette intégration du concept de capabilité s'opère par un déplacement important de son usage initial par Sen ou Nussbaum, à travers un intérêt marqué pour les appropriations ${ }^{42}$ des droits sociaux, telles qu'elles peuvent exister, ou non, dans les diverses situations dans lesquelles les acteurs évoluent. Une telle interrogation conceptuelle peut mettre en évidence le rôle des situations concrètes de vie et de travail dans lesquelles il est, ou non, possible pour les acteurs de percevoir et de mobiliser les actifs sociaux (droits sociaux, aides) mis à leur disposition par les institutions publiques. Cet usage du concept de capabilité permet de mettre à l'épreuve les institutions de l'État social, en particulier les instruments de responsabilisation en interrogeant sur les possibilités réelles des acteurs de faire usage des actifs sociaux, que cela soit un droit social (droit à la formation, droit au congé parental etc.) ou une ressource financière. Les réformes actuelles et passées du système de l'État-providence perpétuent-elles et accentuent-elles vraiment la flexibilité et l'individualité ou bien ne restreignent-elles pas encore plus les possibilités d'action et les libertés individuelles en démantelant dans un processus de plus en plus rapide les procédés établis en matière de sécurité sociale et de transferts intergénérationnels ? ${ }^{43}$ (voir aussi le projet de recherche de Francesco Laruffa au sujet des « critères d'acceptabilité » du travail salarié). Le groupe de travail État-social portera son regard surtout sur les acteurs et leurs rapports aux nouveaux procédés et systèmes de classement bureaucratiques. Une lecture pragmatique issue des sciences sociales du concept de capabilité promet de faire progresser la connaissance tant sur les 
conditions que sur les effets de l'interaction entre bureaucratie et sujet en matière d'Étatprovidence en Europe.

\section{NOTES}

1. Hartmut Kaelble, Das europäische Sozialmodell. Auf dem Weg zum transnationalen Sozialstaat, Berlin : Sigma, 2004. Berthold Vogel, "Der sorgende Staat - ein Kriegsprodukt?", in : Bernd Greiner/Tim Müller/Klaas Voß (dir.), Erbe des kalten Krieges, Hamburg: HIS, 2013, p. 401-412. Phillippe Bezès, Réinventer l'État. Les réformes de l'administration française 1962-2008, Paris : PUF, 2009.

2. Robert Castel, Les Métamorphoses de la question sociale. Une chronique du salariat, Paris : Gallimard, 1995.

3. Wolfgang Streeck, Gekaufte Zeit. Die vertagte Krise des demokratischen Kapitalismus, Berlin: Suhrkamp, 2013. Daniel Gaxie, Nicolas Hubé, Jay Rowell (dir.), L'Europe des Européens. Enquête comparative sur les perceptions de l'Europe. Paris : Economica, 2011.

4. Gosta Esping-Andersen, Les Trois Mondes de l'État-providence. Essai sur le capitalisme moderne, Paris : PUF, 1999. Göran Therborn/Andreas Wirthensohn, Die Gesellschaften Europas 1945-2000. Ein soziologischer Vergleich, Frankfurt am Main : Campus Verlag, 2000.

5. Daniel Rodgers, Atlantic Crossings. Social Politics in a Progressive Age, Cambridge, MA : Belknap Press of Harvard University Press, 1998. Gabriele Metzler/Daniel Letwin, « Sozialstaat. Wohlfahrt und Verteilung » in : Christof Mauch/Kiran K. Patel (dir.), Wettlauf um die Moderne. Die USA und Deutschland 1890 bis heute, München : Pantheon Verlag, 2008, p. 363-392. Bénédicte Zimmermann/ Michael Werner, «Vergleich, Transfer, Verflechtung. Der Ansatz der Histoire croisée und die Herausforderung des Transnationalen ", Geschichte und Gesellschaft, n² 28, 2002, p. 607-636.

6. Wolfgang Streeck/Kathleen Thelen (dir.), Beyond Continuity. Institutional Change in Advanced Political Economies, Oxford/New York : Oxford University Press, 2005, p. 19.

7. Bénédicte Zimmermann, «Pragmatism and the Capability Approach: Challenges in Social Theory and Empirical Research ", European Journal of Social Theory, n 9, 2006, p. 467-484.

8. Christoph Conrad, Vom Greis zum Rentner. Der Strukturwandel des Alters in Deutschland zwischen 1830 und 1930, Göttingen : Vandenhoeck \& Ruprecht, 1994. Gerd Hardach, Der Generationenvertrag. Lebenslauf und Lebenseinkommen in Deutschland in zwei Jahrhunderten, Berlin : Duncker \& Humblot, 2006. Joerg Tremmel, A Theory of Intergenerational Justice, London: Sterling, 2009. Siegfried Blasche/Diether Döring (dir.), Sozialpolitik und Gerechtigkeit, Frankfurt a.M. : Campus, 1998. Ohad Parnes/Ulrike Vedder/Stefan Willer., Das Konzept der Generation. Eine Wissenschafts- und Kulturgeschichte, Frankfurt am Main : Suhrkamp, 2008.

9. Christina May, Generation als Argument. Konflikte um die Rentenversicherung in Deutschland, Großbritannien und den Niederlanden, Frankfurt am Main : Campus, 2010.

10. Louis Chauvel, Le Destin des générations. Structure sociale et cohortes en France du XXe siècle aux années 2010, Paris: PUF 2010. Axel Grosseries, Penser la justice entre les générations. De l'affaire Perruche à la réforme des retraites, Paris : Aubier, 2004.

11. Berthold Vogel/Jens Kersten/Claudia Neu, Demografie und Demokratie. Zur Politisierung des Wohlfahrtsstaates, Hamburg : HIS, 2012.

12. Arnaud Lechevalier/Yves Ullmo, «La réforme de la protection sociale du risque dépendance. Les enjeux économiques et sociaux », Revue de l'OFCE, n 77, 2001, p. 157-198. 
13. Bénédicte Zimmermann, "Pragmatism and the Capability Approach: Challenges in Social Theory and Empirical Research », European Journal of Social Theory, n 9, 2006, p. 467-484.

14. Gabriele Metzler, Der deutsche Sozialstaat. Vom Bismarck'schen Erfolgsmodell zum Pflegefall, Stuttgart/München : Springer, 2003.

15. Hartmut Kaelble, Sozialgeschichte Europas. 1945 bis zur Gegenwart, Bonn: bpb, 2007. Pierre Rosanvallon, La Société des égaux, Paris: Seuil, 2012. Serge Paugam/François-Xavier Schweyer, «Les inflexions de l'État social. Vers un nouveau régime?», in: Olivier Galland/ Yannick Lemel (dir.), La Société française. Un bilan sociologique des évolutions depuis l'après-guerre, Paris : Armand Colin, 2011, p. 227-252.

16. Thomas Mergel, «Gleichheit und Ungleichheit als zeithistorisches und soziologisches Problem », Zeithistorische Forschungen/Studies in Contemporary History, $\mathrm{n}^{\circ} 10 / 2,2013$, URL : http:// www.zeithistorische-forschungen.de/16126041-Mergel-2-2013 (lien vérifié le 1er décembre 2014). 17. Pierre Rosanvallon, La Société des égaux, Paris : Seuil, 2012.

18. Claus Offe, «Politische Herrschaft und Klassenstrukturen. Zur Analyse spätkapitalistischer Gesellschaft », in : Gisela Kress/Dieter Senghaas (dir.), Politikwissenschaft. Eine Einführung in ihre Probleme, Frankfurt am Main : Fischer, 1969, p. 155-189. Hartmut Kaelble, Industrialisierung und soziale Ungleichheit, Göttingen: Vandenhoeck \& Ruprecht 1998 (1983). Hans-Ulrich Wehler, Die neue Umverteilung. Soziale Ungleichheit in Deutschland, München : Beck, 2013.

19. Patrick Emmenegger/Silka Häusermann/Bruno Palier (dir.), The Age of Dualization. The Changing Face of Inequality in Deindustrializing Societies, Oxford/New York : Oxford University Press, 2012.

20. Christian Topalov, Naissance du chômeur 1880-1910, Paris: Albin Michel, 1994. Bénédicte Zimmermann (dir.), Arbeit und Nationalstaat. Frankreich und Deutschland in europäischer Perspektive, Frankfurt am Main: Campus, 2000. Delphine Serre, Les Coulisses de l'État social. Enquête sur les signalements d'enfant en danger, Paris : Raisons d'agir, 2009.

21. Lutz Raphael, « Die Verwissenschaftlichung des Sozialen als methodische und konzeptionelle Herausforderung für eine Sozialgeschichte des 20. Jahrhunderts », Geschichte und Gesellschaft, n - 22/2, 1995, p. 165-193.

22. Hans Günther Hockerts/Hans Günter (dir.), Drei Wege deutscher Sozialstaatlichkeit. NS-Diktatur, Bundesrepublik und DDR im Vergleich, München: Oldenbourg Wissenschaftsverlag, 1998. Thomas Etzemüller (dir.), Die Ordnung der Moderne. Social Engineering im 20. Jahrhundert, Bielefeld: transcript, 2009. Eric Engstrom/Volker Hess/Ulrike Thoms (dir.), Figurationen des Experten: Ambivalenzen der wissenschaftlichen Expertise im ausgehenden 18. und frühen 19. Jahrhundert, Frankfurt am Main : Peter Lang, 2005.

23. Lutz Raphael/Heinz E. Tenorth (dir.), Ideen als gesellschaftliche Gestaltungskraft im Europa der Neuzeit : Beiträge für eine erneuerte Geistesgeschichte, München : Oldenbourg, 2006.

24. Didier Georgakakis/Marine de Lassalle, "Genèse et structure d'un capital institutionnel européen ", Actes de la recherche en sciences sociales, $n^{\circ}$ 166-167, 2007, p. 38-53. Didier Georgakakis, Le Champ de l'Eurocratie - Une sociologie politique du personnel de l'UE, Paris : Economica, 2012.

25. Hélène Michel, «Le syndicalisme dans la 'gouvernance' européenne. Formes de représentation et pratiques de défense des intérêts sociaux en questions ", Politique européenne, $\mathrm{n}$ - 27, 2009, 27, p. 129-152. Anne-Catherine Wagner, «Les syndicalistes de la Confédération européenne des Syndicats. Un capital européen spécifique?» in : Didier Georgakakis (dir.), Le Champ de l'Eurocratie. Une sociologie politique du personnel de l'Union européenne, Paris : Economica 2012, p. 241-256

26. Sandrine Kott, L'État social allemand. Représentations et pratiques, Paris : Belin, 1995.

27. Luc Boltanski, Les Cadres. La formation d'un groupe social, Paris : Minuit 1982. Alain Desrosières, Die Politik der großen Zahlen. Eine Geschichte der statistischen Denkweise, Berlin: Springer Verlag, 2004. 
28. Jay Rowell/Etienne Pénissat, « Note de recherche sur la fabrique de la nomenclature socioéconomique européenne $\mathrm{ESeC} »$, Actes de la recherche en science sociale, $\mathrm{n}^{\circ}$ 191-192, 2012/1-2, p. 126-135.

29. Robert Castel, Les Métamorphoses de la question sociale. Une chronique du salariat, Paris: Gallimard, 1995.

30. Edward Thompson, The Making of the English Working Class, London : Victor Gollancz, 1963. Peter Wagner/Claude Didry/Bénédicte Zimmermann (dir.), Le Travail et la nation. Histoire croisée de la France et de l'Allemagne, Paris: Editions Maison des Sciences de l'Homme, 1999. Thomas Welskopp, Das Banner der Brüderlichkeit: Die deutsche Sozialdemokratie vom Vormärz bis zum Sozialistengesetz, Bonn : Dietz, 2000.

31. Robert Castel, Les Métamorphoses de la question sociale. Une chronique du salariat, Paris: Gallimard, 1995. Serge Paugam (dir.), L'Exclusion, l'état des savoirs, Paris : La Découverte, coll. «Textes à l'appui », 1996.

32. Vincent Dubois, «État social actif et contrôle des chômeurs. Un tournant rigoriste entre tendances européennes et logiques nationales », in : Didier Chabanet/Jean Faniel (dir.), L'Europe du chômage, Paris : L'Harmattan, 2007, p. 73-97.

33. Bénédicte Zimmermann, La Constitution du chômage en Allemagne. Entre professions et territoires, Paris : Editions de la MSH, 2001. Ead., «Pragmatism and the Capability Approach : Challenges in Social Theory and Empirical Research », European Journal of Social Theory, n 9, 2006, p. 467-484.

34. Kiran Patel/Ulrike von Hirschhausen, «Europeanization in History. An Introduction » in: Martin Conway, Kiran Patel (dir.), Europeanization in the Twentieth Century. Historical Approaches, Basingstoke : Palgrave Macmillan, 2010, p. 1-18.

35. Wolfram Lamping, " Auf dem Weg zu einem postnationalen Sozialstaat ? Die Sozialpolitik der Europäischen Union» in: Klaus Schubert (dir.), Europäische Wohlfahrtssysteme. Ein Handbuch, Wiesbaden : Verlag für Sozialwissenschaften, 2008, p. 595-620.

36. Sebastian Buettner, Mobilizing Regions - Mobilizing Europe. Expert Knowledge and Scientific Planning in European Regional Development, London : Routledge, 2014. Alexander Nützenadel, Stunde der Ökonomen. Wissenschaft, Politik und Expertenkultur in der Bundesrepublik. 1949-1974, Göttingen : Vandenhoeck \& Ruprecht, 2005.

37. Nikola Tietze/Monika Eigmüller, « Socio-histoire der Europäisierung. Sozialpolitik, rechtliche Gleichbehandlung und öffentliche Dienstleistung im Wirbel europäischer Maßstäbe », 2012 : http://www.his-online.de/forschung/nation-und-gesellschaft/1864/.

38. Yves Déloye, Sociologie historique du politique, Paris : La Découverte 2007. Gérard Noiriel, La Sociohistoire, Paris : La Découverte, 2006. Gilles Pollet, « Sociogenèse de l'État social français. La société civile contre - ou tout contre - l'État?», in: Michèle Dupré/Olivier Giraud/Michel Lallemand (dir.), Trajectoires des modèles nationaux - État, démocratie et travail en France et en Allemagne, Bruxelles: Peter Lang, 2013, p. 103-117. Robert Salais/Nicolas Baverez/Bénédicte Reynaud, L'Invention du chômage, Paris : PUF, 1986. Bénédicte Zimmermann, « Pragmatism and the Capability Approach: Challenges in Social Theory and Empirical Research », European Journal of Social Theory, n 9, 2006, p. 467-484.

39. Peter Berger/Thomas Luckman, The Social Construction of Reality: A Treatise in the Sociology of Knowledge, Garden City, NY : Doubleday \& Company Inc, 1966.

40. Amartya Sen, Capability and well-being, in : Amartya Sen/Martha Nussbaum (dir.), The Quality of Life, Oxford: Oxford University Press, 1993, p. 31-53. Amartya Sen, Commodities and Capabilities, Amsterdam : North-Holland, 1985. Amartya Sen, «Equality of What? » in : Amartya Sen (dir.), Choice, Welfare and Measurement, Oxford : Blackwell, 1982, p. 353-369.

41. Bénédicte Zimmermann, "Pragmatism and the Capability Approach: Challenges in Social Theory and Empirical Research", European Journal of Social Theory, 2006, p. 467-484. Jean de Munck/Bénédicte Zimmermann, La Liberté au prisme des capacités. Amartya Sen au-delà du libéralisme, Paris : Editions de l'EHESS, 2008. 
42. Jay Rowell, Le Totalitarisme au concret. Les politiques du logement en RDA, Paris : Economica, 2006. 43. Neil Gilbert, Transformation of the Welfare State: The Silent Surrender of Public Responsibility, Oxford : Oxford University Press, 2002.

\section{AUTEURS}

KARIM FERTIKH

(EHESS, Paris)

\section{HEIKE WIETERS}

(Université Humboldt, Berlin) 